\title{
Ring-shaped bifocal lens used for fluorescent self-referenced holographic imaging
}

\author{
Márton Zsolt Kiss ${ }^{1,2}$
}

\begin{abstract}
We propose an alternative and simple solution to self-referenced digital holographic imaging based on a ring-shaped bifocal lens, without the need of any mirrors, polarizers or spatial light modulators. We discuss the imaging properties of the ring-shaped bifocal lens in self-referenced holography. The easy applicability of this bifocal lens is demonstrated on a realized microscope setup for volumetric observation of freely moving fluorescent objects, based on a conventional light microscope.
\end{abstract}

Keywords: Digital holography, Fluorescence microscopy, Holographic optical elements, Vision in depth, Single-shot imaging

\section{Background}

The aim of our research is to develop a microscope that can detect, localise and image freely moving fluorescent objects within a thick volume in real time. The realization of such an instrument would result in an immediate industrial benefit, for example, real-time water monitoring systems [1] can be built, where the use of self-referenced holography $[2,3]$ is undoubtedly very profitable.

Holographic imaging is based on an interference phenomenon, in which wavefronts are captured to reconstruct the image of the measured objects [4]. Two of its main advantages are the possibility to increase the depth of the observed volume without having a considerable loss in the resolution [5], and the potential of lens-less imaging [6]. In the traditional in-line [7] and off-axis [8] setups, one light source with an appropriate length of coherence is used to implement both the target and the reference beams. Such a light source can be a laser [9], a LED [10], an electron gun [11], or some other sources [12] as well.

However, in some cases, these traditional arrangements cannot be applied. Either the size and/or the

\footnotetext{
Correspondence: kiss.marton@sztaki.mta.hu

${ }^{1}$ Faculty of Information Technology and Bionics, Pázmány Péter Catholic University, Budapest $\mathrm{H}-1083$, Hungary

${ }^{2}$ Hungarian Academy of Sciences, Institute for Computer Science and Control, Computational Optical Sensing and Processing Laboratory, Budapest $\mathrm{H}-1111$, Hungary
}

position of the target object does not permit the proper interference of the target and reference beams, or the light emission of the targets themselves is the relevant feature that is to be detected and reconstructed. In these cases, Self-referenced Holographic Setups (SHS) can be applied. In these setups, the target and reference beams are the differently modulated lights of the same emitted (or reflected) light of the measured object. The requirement of proper interference is that the optical path difference of the two beams has to be smaller than the coherence length of the light.

Self-referenced Holography (SH) is frequently applied to image fluorescent [13], distant and extended objects [14], or stars [15]. An architecture of the SHS can be based on either an interferometer (e.g. Hariharan-Sen $[16,17])$, bi- or multifocal lens $[18,19]$, or on a diffractive optical element [20].

Although the concept of bifocal lens based SHS was already presented in the early years of holography [4], and the application of birefringent bifocal lens, double half lens, Fresnel zone plate, and their mixed solutions were proposed, only some of them were tested experimentally [21]. Recently, the application of Spatial Light Modulators (SLM) extended the list of bi- and multifocal lens based SHS [22].

Usually, interferometer-based SHSs are quite large and complex, due to the mirrors required, which also makes 
them exceptionally sensitive to vibrations. Furthermore, the used beam splitter typically result in more than $50 \%$ light intensity loss. Polariser based SHSs are also doomed to face the latter problem, which is also a particularly challenging limitation when the light emission of the objects is little, as in the case of the abovementioned fluorescent imaging.

All the SLM- and some of the interferometer-based setups also offer the possibility of twin-image elimination; however this can usually be fulfilled only for static objects, as the method is based on phase-shifting interferometry and requires multiple exposures of the same object [23].

The above limitations of the earlier approaches directed our attention to the application of a special bifocal lens in SHS. That is, to apply a Ring-shaped Bifocal Lens (RBL) [24] to implement SH. According to our best knowledge, so far this approach was not proposed, used or tested by others.

In this paper, our goal is to prove that the RBL is an efficient tool for making holography based fluorescent volume detection, localization and imaging. First, let us overview the main details of the imaging method of the $\mathrm{SH}$, and outline the hologram generating principle of the RBL in the next section. Second, the main advantages and disadvantages of the RBL made holograms are discussed. Finally, we demonstrate the applicability, and the ability to fluorescent targets volume imaging of the RBL through the experimental study of the RBL based SHS.

\section{Methods}

\section{The self-referenced imaging and the RBL}

Let us briefly summarize the self-referenced hologram generating method. An SHS generates two waves (a wave pair) with different wavefront curvatures from a light coming from a point of the object.

The interference of the pair of waves produces a Selfreferenced Interference Pattern (SIP). The SIP captured by a digital camera is the digital intensity hologram. Several object points will have several SIPs by the SHS, and the camera captured image of their sum also is the digital intensity hologram.

When the light coming from different points of the object are coherent with each other, the complex amplitude of their SHS generated SIPs is added. Otherwise, only the intensities of their SIPs are summed. (Obviously there is a case when the SIPs are partially coherent with each other.) Thus, in the particular case of fluorescent objects, only the intensities of the SIPs are summed, irrespectively of the type of their excitation light.

Let us analyse the formation of a single SIP in the following. A spherical wave emitted (or reflected) by a single point is divided than modulated differently by the SHS into two spherical waves, with a radius of curvatures $\left(R_{1}, R_{2}\right)$ at the detector plane. Our calculations pointed out that the interaction of these waves generates an interference intensity pattern, which is the same as the intensity of the interference of a plane wave and a spherical wave with radius of curvature $R_{d}$ (Eq. (1)), where

$$
\mathrm{R}_{d}= \pm \frac{\mathrm{R}_{1} * \mathrm{R}_{2}}{\mathrm{R}_{1}-\mathrm{R}_{2}}
$$

$R_{d}$ is used to define the reconstruction distance of the corresponding object point. As the SIPs are incoherently summed up, they do not disturb each other, and therefore, their reconstruction distances remain unbiased. This property is important when the reconstruction is used for object localization. However, the more incoherent summation of the SIPs, the less relative dynamic range of the detector, which results in considerable loss of contrast of the captured digital hologram.

Next, our novel optical solution is presented for creating double coherent waves from a single one for the purpose of self-referenced holography.

\section{RBL hologram generation}

At the central point of our SHS stands the Ring-shaped Bifocal Lens that realise the self-referenced hologram with the required beam splitting. This splitting is the result of the division of the aperture of the RBL. The two apertures are different; the central one is circular while the other is a ring around it. Both of them are symmetric to the optical axis. These aperture areas have a focus difference, which in general can be reached with the optical property of the material (e.g. grin lens), or with the geometry. Here, we use an RBL where the geometry of the lens generates the different focuses. To ensure exactly two focuses, only one of the two surfaces (the right one) is diversified, as it can be seen in Fig. 1.

In our experiments, we use a custom made RBL, which consists of a central plano-convex lens (focal length $400 \mathrm{~mm}$ ), and a "biplane ring-shaped lens" having infinite focus. The outer diameter of the whole RBL is $10 \mathrm{~mm}$ while the diameter of the inner lens is $6 \mathrm{~mm}$. The scheme of the hologram generation of the actual RBL is shown in Fig. 2 when the source object is at an infinite distance. One can see that the RBL creates two beams, a central placed cone shaped one, and a hollow one. These beams have a ring-shaped cross section at the plane of detection. The self-referenced hologram of a single point created by the RBL is ring-shaped as it is shown in Fig. 4. Because the middle of this hologram is missing, we call it gappy hologram. The shape of the hologram is determined by the shape of the outer aperture of the RBL, and only the divergences of the beams depend on the focal parameters (Fig. 2). 


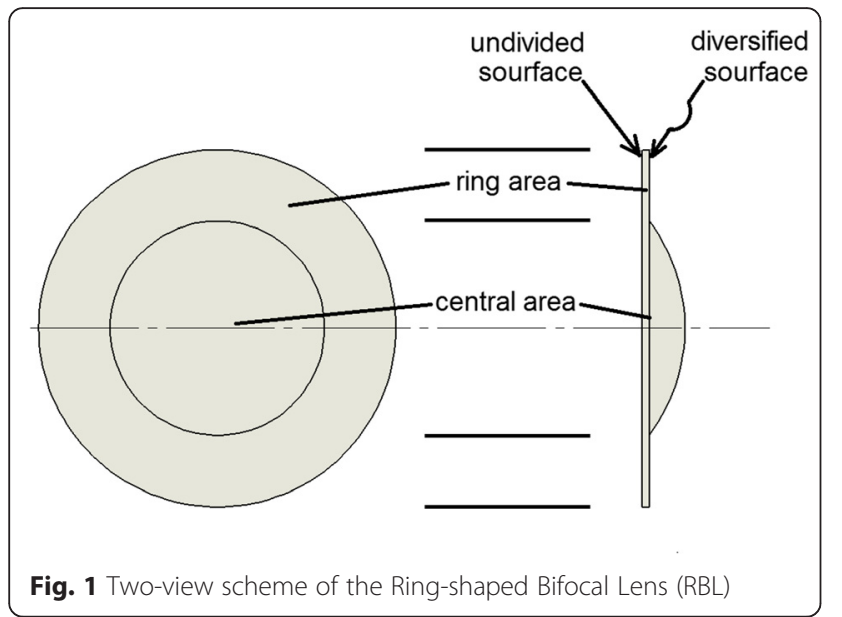

The RBL modulates the incident $\mathrm{U}(\mathrm{r}, \mathrm{RBL} 1)$ complex amplitude beam the following way (Eq. (2)):

$$
U(r, R B L 2)=U(r, R B L 1) *\left(A_{o} L_{o}+A_{c} L_{c}\right)
$$

Where $\mathrm{U}(\mathrm{r}, \mathrm{RBL2})$ denotes the generated beam, $\mathrm{A}_{\mathrm{o}}$ (Eq. (3)) and $A_{c}$ (Eq. (4)) correspond to the ring and the central apertures of the RBL, while $\mathrm{L}_{\mathrm{o}}$ (Eq. (5)) and $\mathrm{L}_{\mathrm{c}}$ (Eq. (6)) describe the phase modulation property of the lenses in the ring-shaped and the central area.

$$
\begin{aligned}
& A_{o}=\operatorname{sign}(r-R) \\
& A_{c}=\operatorname{sign}(R-r)
\end{aligned}
$$

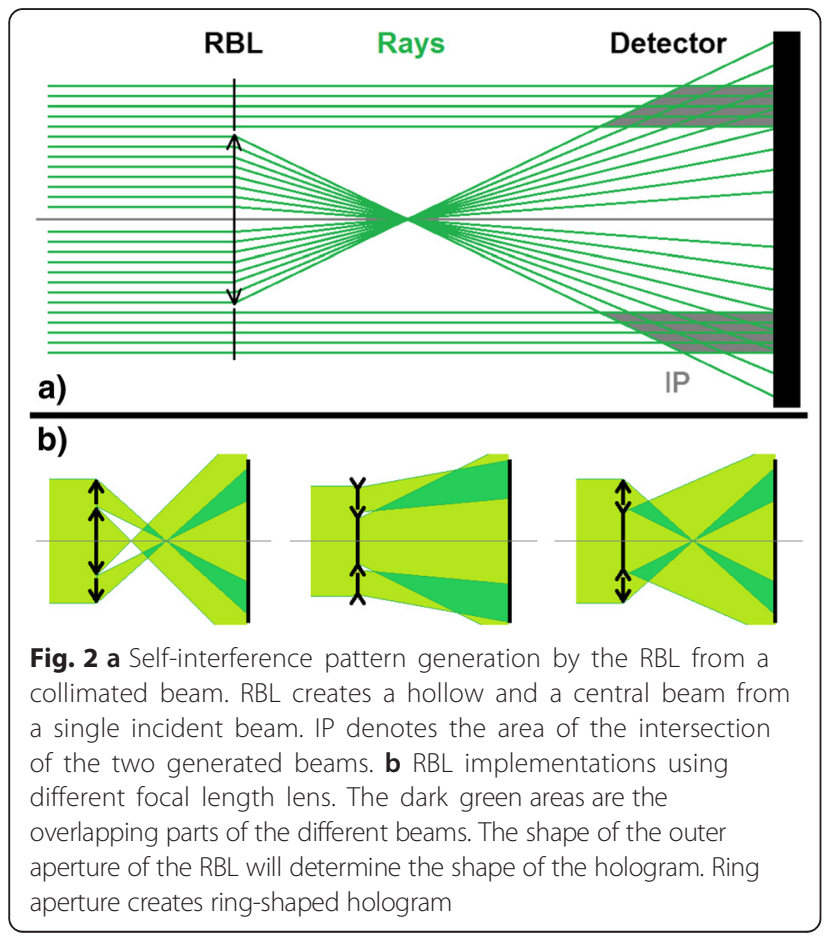

$$
\begin{aligned}
& L_{o}=\exp \left(i \pi /\left(\lambda * f_{o}\right) r^{2}\right. \\
& L_{c}=\exp \left(i \pi /\left(\lambda * f_{c}\right) r^{2}\right.
\end{aligned}
$$

where

$$
\operatorname{sign}(x)=\left\{\begin{array}{l}
0, \quad x<0 \\
1, \quad x \geq 0
\end{array}\right.
$$

and $\mathrm{r}$ denotes the distance from the optical axis, $\mathrm{R}$ the inner radius of the RBL, and $\lambda$ the applied wavelength, while $f_{o}$ and $f_{c}$ are the focal lengths of the ring and the central areas, respectively.

\section{The optical path difference problem}

It is known, that in SHSs the Optical Path Difference (OPD) between their different optical ways have to be smaller than the coherent length of the used light to have interference phenomena and hologram.

First, let us observe the OPD on a convenient Hariharan-Sen [15] interferometer based SHS in the case when a single target point is on the optical axis. It is also shown in Fig. 3, and one can see, that even if the light of that target point is split, modulated independently than united to make interference, the created two beams after their union have zero OPD along the optical axis. Due to the curvature differences of the wavefronts of the two

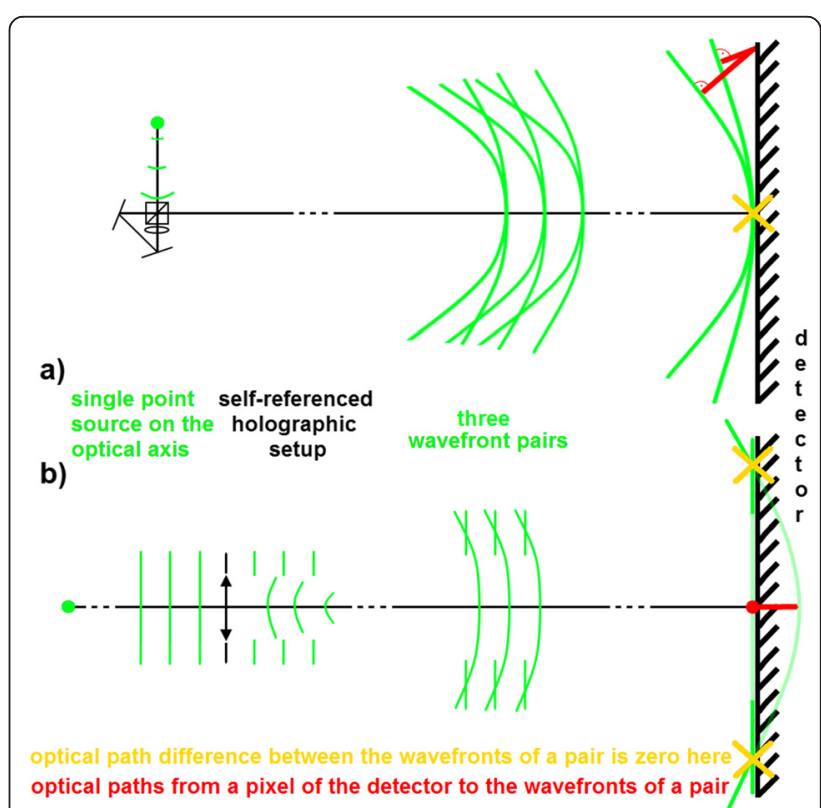

Fig. 3 a Wavefronts of a single-point source modulated by a Hariharan-Sen interferometer. $\mathbf{b}$ Wavefronts of a single-point source modulated by the RBL. At the detector plane, the yellow crosses denote the places, where the OPD between the two beams is zero, thus we have a constructive interference. The red lines (or dot that is a zero length line) originating from a pixel of the detector show the optical paths between the pixel and the pair of wavefronts. The length difference between such red lines is the OPD at the pixel in question 
beams their OPD increases with their distance from the optical axis. At the plane of the detector, where the OPD become bigger than the coherent length of the used light the interference phenomena will disappear, and there will be the border of the hologram.

Considering these findings and that the RBL formed hologram is a gappy one, we aimed to design the RBL to achieve zero OPD between the central beam and the hollow beam at the common ring-shaped area at the detector plane not on the optical axis. We illustrate the design on Fig. 3. To have this zero OPD we calculated the required thickness difference between the central and the ring area of the custom made RBL.

As the relative curvatures between the wavefronts change during the propagation, it can be seen that the OPDs at the plane of detection also depend on the actual position of the detector.

\section{The attributes of the RBL}

Using an RBL based SHS a single point target generates a ring-shaped (gappy) hologram, which is shown in Fig. 4. In the central region of such hologram, the interference pattern with the low spatial frequency components is missing because the intensity of the hollow beam is zero at that place.

The reconstructed image of the ring-shaped hologram will appear in the middle of the ring, not on the interference fringes. Thus, an even (homogenous) background can have the reconstructed image. Furthermore, the twin image diffractions expanding outwards and never overlap with the image reconstruction itself. However, we should note, that the absence of low-frequency components might result in deterioration of the image.

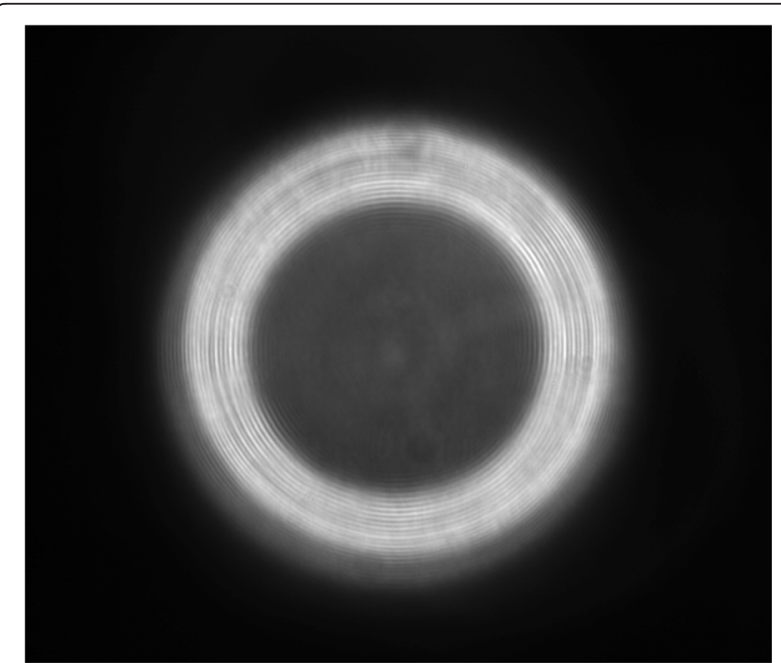

Fig. 4 A typical self-referenced digital intensity hologram using an RBL. Interference fringes only appear in the ring area, due to the geometry of the hollow beam. This results in that the low spatial frequency components will be not recorded in the hologram, and this way they will be missing from the hologram reconstructions too
Even if the image reconstruction is not perfect, we will show that using the proposed method, one can still detect the 3D position of the target objects and will be able to discriminate between them correctly.

Using numerical simulations, we investigated the relationship between the parameters of the ring-shaped hologram and its reconstruction properties. We handled both the depth of focus of the reconstruction and the Strehl ratio (the quotient of the maximum intensities of an actual and a reference reconstruction images) with exceptional care.

First, we simulated the diffraction limited hologram of a single point source. In the simulated case, that contains seventeen concentric rings of interference. Second, starting from the middle one, we mask more and more interference rings, thus simulating gappy holograms. The relationship between the number of missing interference rings and the quality of the reconstructed images were measured.

The reconstruction distance was set $3000 \mu \mathrm{m}$ while the wavelength and pixel size were $530 \mathrm{~nm}$, and $0.9 \mu \mathrm{m}$ respectively. The simulated holograms, the reconstructions and the measured parameters like the Strehl ratio and the depth of focus are shown in Fig. 5.

To determine the depth of focus I used the following definition: a point is reconstructed at a special reconstruction distance if the intensity of the reconstructed point is not lower than the maximal intensity of its background. The background intensity is estimated from a

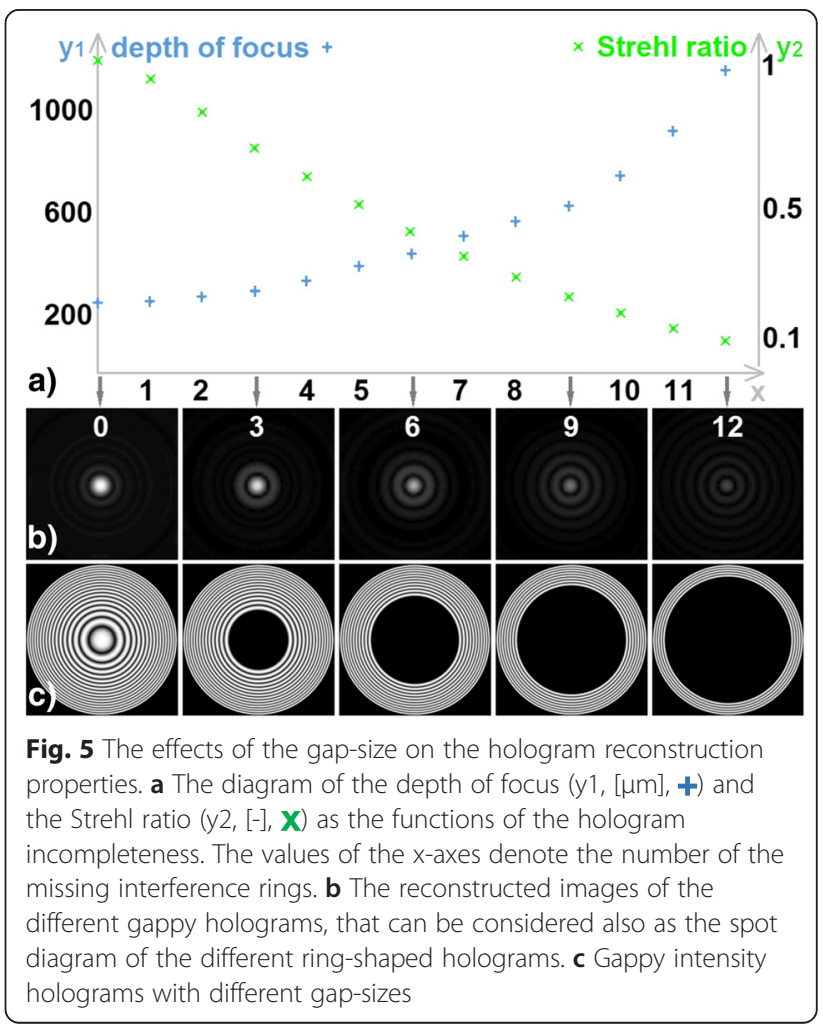


surrounding area nine times bigger than the reconstructed point.

The Strehl ratio is defined as the quotient of the maximum intensities of the actual gappy hologram reconstruction and the full hologram reconstruction.

Our results show that by the increase of the missing area, the Strehl ratio decreases and the depth of focus increases. It can also be observed that the depth of focus is increasing when a ring-shaped hologram becomes thinner, but this effect is only apparent when the gap is sufficiently large, in the first few cases when the gap is small, it is unnoticeable. Thus, setting the rate of the aperture areas of the hologram with the aperture parameters of the RBL, these properties of the reconstructed image can be hold. Observing the point spread functions (PSF) of the spot diagram, it can be seen that as the size of the central spot and its maximal intensity decrease while the artefact caused by the surrounding ring increase with the increase of the relative gap size. As these diffraction rings can produce interference with the fringes of the target object reconstruction, the size and shape (spot, line, grid...) parameters of the target will also shape the resolution of the imaging. In section 4.2 the PSF of the built SHS setup is presented.

We have to note that the narrow ring-shaped hologram of a single point source has an elongated, line-like image along the direction of the reconstruction. This is an axicon-like property. The further discussion is of this topic is beyond the scope of the present paper.

\section{The self-referenced holographic microscope}

In this section, we discuss an actual implementation of a self-referenced digital holographic microscope based on a commercially available microscope and the RBL discussed so far.

For this purpose, the RBL was placed into an Olympus (IX71) microscope, on the rear surface of the objective (Olympus, 4x Plan, NA $=0.16, \mathrm{f}=45 \mathrm{~mm}$, infinity corrected). The hologram was recorded with a high sensitivity C-MOS camera (ASI120MM-S, 1280x960). The camera was placed $40 \mathrm{~mm}$ out of the focal plane of the camera adapter (U-TV0.5XC-3) which was placed on a trinocular tube (U-TR30-2). The excitation light had a wavelength of $405 \mathrm{~nm}$. For the observance of the fluorescent USAF testtarget (EO Stock No. 57-792), a dichroic emission filter was applied (10 $\mathrm{nm}$ bandwidth and the central wavelength is $530 \mathrm{~nm}$, Thorlabs FB530-10) between the RBL and the trinocular tube. When the algae sample was measured, we also used a longpass filter (Thorlabs FEL 600). The scheme of the measuring setup is illustrated in Fig. 6.

As the RBL generates two different beams from the light of a single point object, it also generates its two real images at the first and the second image planes. The first image is produced by the cone-shaped beam, which is surrounded by the defocused image of the hollow beam. On the other hand, the second image is reproduced from the hollow beam, while the defocused cone shaped beam overlaps with it. The two images appear at different planes. We illustrate such dual-imaging at Fig. 7.

\section{Results}

\section{Imaging with the RBL basedself-referenced} holographic microscope

The angular spectrum method [25] was applied to reconstruct the objects from their digital holograms. Three different measurements were made to test the operation and efficiency of the introduced SHS.

In the first one, holograms of different size of objects from the same depth were captured and reconstructed to get partial information about the resolution. In the second experiment, the holograms of an object in three different distances were measured and reconstructed to know that the setup can make volume detection. Finally, in the third measurement, auto-fluorescent self-referenced holographic image of an algae sample was captured and evaluated to realise that it can make auto-fluorescent biological measurements.

\section{The effect of the object size on the reconstruction quality}

Our first test consisted of using four different samples from the number " 2 " of USAF 1951 fluorescent test target, with different sizes. One by one, we placed these test targets at the same position, just on the focal plane of the objective. One of the four sample reconstructions and the cropped images of the reconstructions are shown in Fig. 8.

Comparing these reconstruction results (Fig. 8), it can be seen that the more point sources contributes to the object the lower contrast the reconstructed image will have,

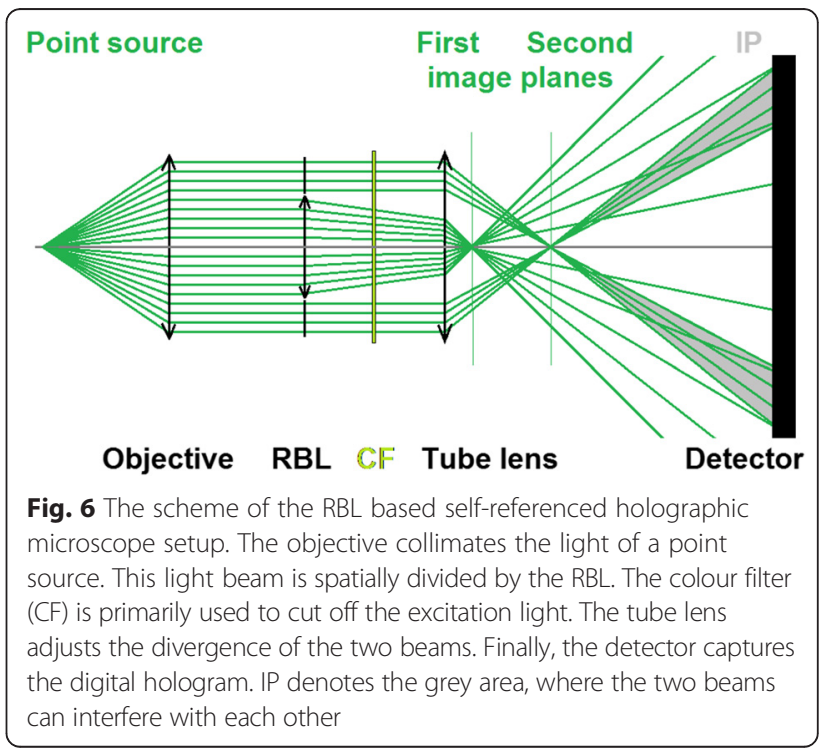


as we discussed at the beginning of section 2. The missing information of the hollow beam limits the resolution of the imaging, but not the resolution of the detection.

\section{Reconstruction of object in different depths}

We estimate the depth of the measurable volume by placing the object in the $-0.5 \mathrm{~mm}, 0 \mathrm{~mm},+1 \mathrm{~mm}$ positions along the optical axis. The position of $0 \mathrm{~mm}$ corresponds to the focal plane of the used objective, and the others are end positions of the adjusting mechanism. We note that the position of the object was also changed in the lateral direction to avoid the overlapping of the reconstructed images. As we used one fluorescent USAF test target, we had to position it

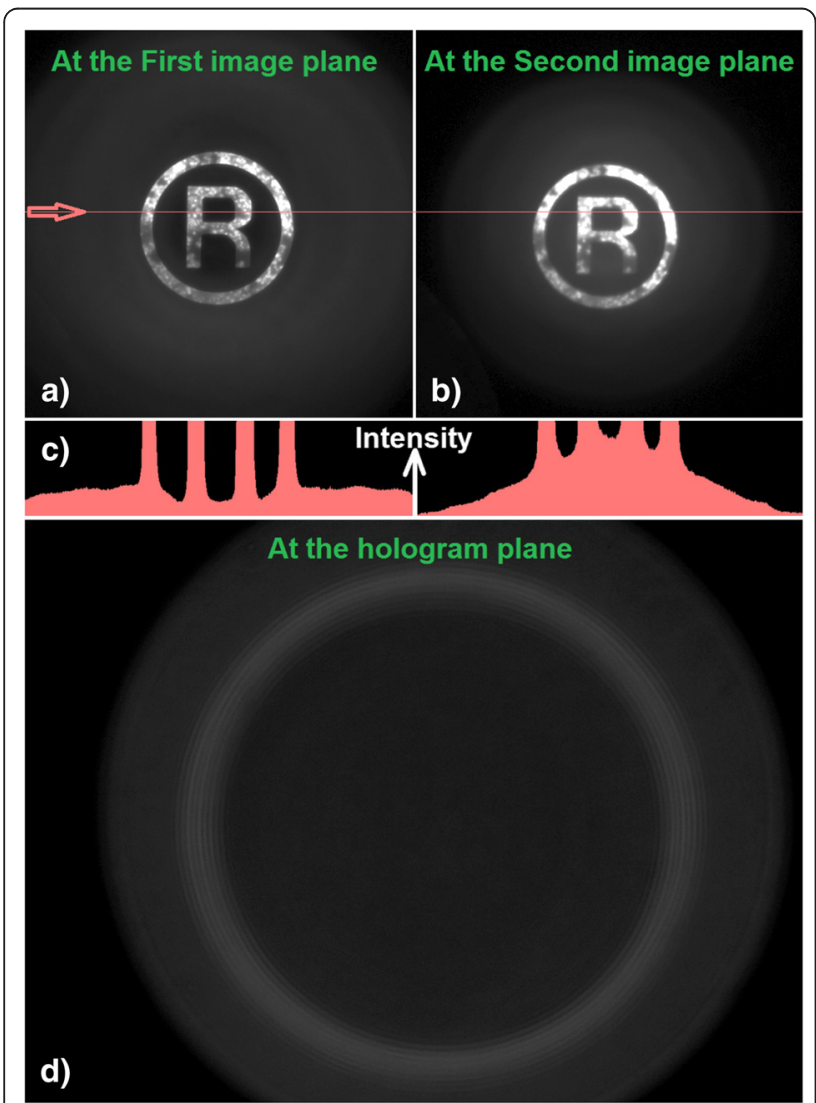

Fig. 7 As the RBL is a bifocal lens, it is a dual-imaging system. It creates double images of one target at different planes. (These planes are also captured.) The first image is produced by the cone-shaped beam, which is surrounded by the defocused image of the hollow beam. On the other hand, the second image can be reproduced from the hollow beam, while the defocused cone shaped beam overlaps with it. Here the two image planes and the hologram plane can be seen with the intensity patterns generated with the RBL based Self-referenced Holographic System (SHS), where the target is the sign " ${ }^{\circledR}$. One can see, that the first image (a) is surrounded by the hollow beam. Vice versa, the second image (b) overlaps with the defocused cone beam. $\mathbf{c}$ is the intensity distribution of the $\mathbf{a}$ and $\mathbf{b}$ images along the red line, and $\mathbf{d}$ is the hologram of the same target and make the exposures three times to capture the three holograms belonging to the different depths.

By adding the intensities of these hologram images, we simulated the recording of a single exposure hologram of the target objects, which are in different depth and lateral positions. In the following, we shall call the result as Measured Hologram (MH). The MH has inhomogeneous background that is caused by the zero order diffractions. This background results in an elevated noise in the reconstructed image. To eliminate the zero order I subtracted the $\mathrm{MH}$ from the sharpened $\mathrm{MH}$, which was created with the "sharpeningFilter" function of Matlab. The sharpening also increased the contrast of the interference fringes, while the change of the zero order was negligible. As the proper estimation of the zero-order term is needed for the correct reconstruction, the eliminated zero-order is replaced by an even unit size intensity in the hologram. We call this modified MH as Corrected Hologram $(\mathrm{CH})$.

In Fig. 9, we show the halves of both of the $\mathrm{MH}$ and the $\mathrm{CH}$ then the reconstructions of the $\mathrm{CH}$ at different depths, and also one $\mathrm{MH}$ reconstruction.

Beside the efficiency of the introduced hologram correction method, the reconstructed images also demonstrate that the RBL based SH microscope can reconstruct an object within a volume having more than a $1.5 \mathrm{~mm}$ depth. Considering the fact that a conventional microscope has only $55 \mu \mathrm{m}$ depth of focus at this magnification level, the

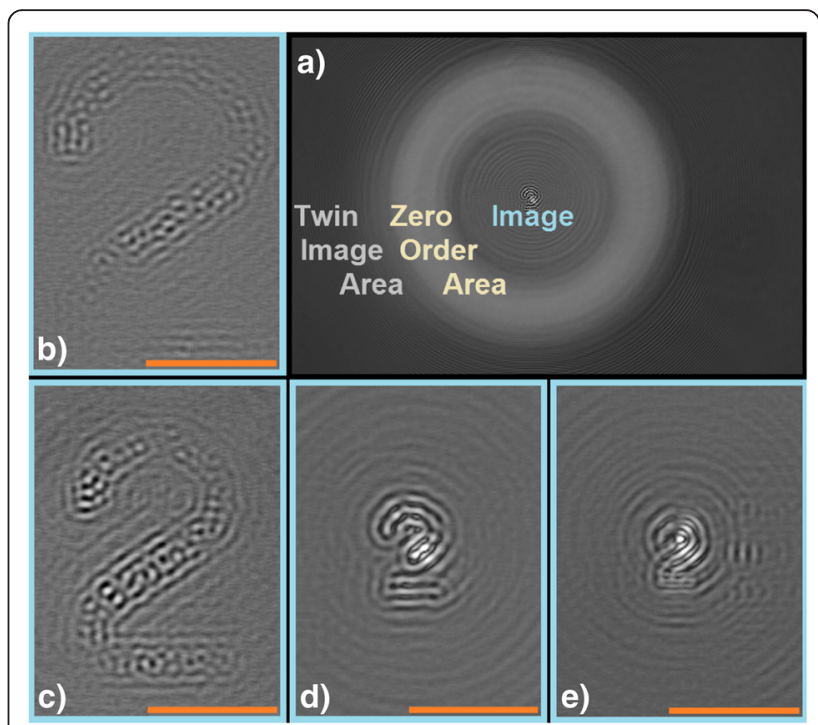

Fig. 8 Resolution and contrast measurements with the RBL based SHS. a One reconstructed hologram; b-e the reconstructed images of different sized "2" shaped objects, where the physical sizes of the test targets were decreasing. The orange scale bars are $200 \mu \mathrm{m}$ long. One can see, that as more point sources contributes to the object the lower contrast the reconstructed image will have. The missing information of the hollow beam limits the resolution of the imaging, but not the resolution of the object detection 


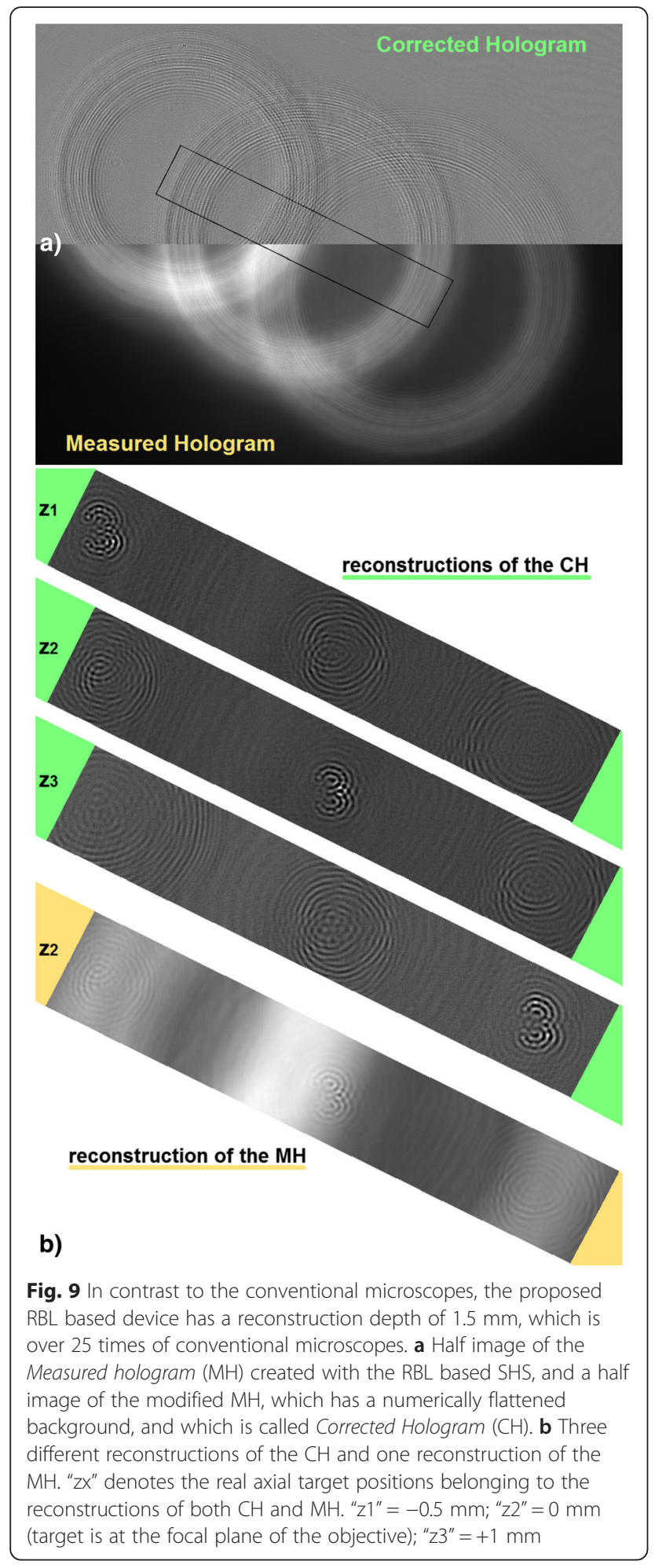

increase enabled by the proposed RBL based microscope is over 25 times.

The PSF characteristics of the discussed RBL based SHS and commercial microscope that belongs to a point source

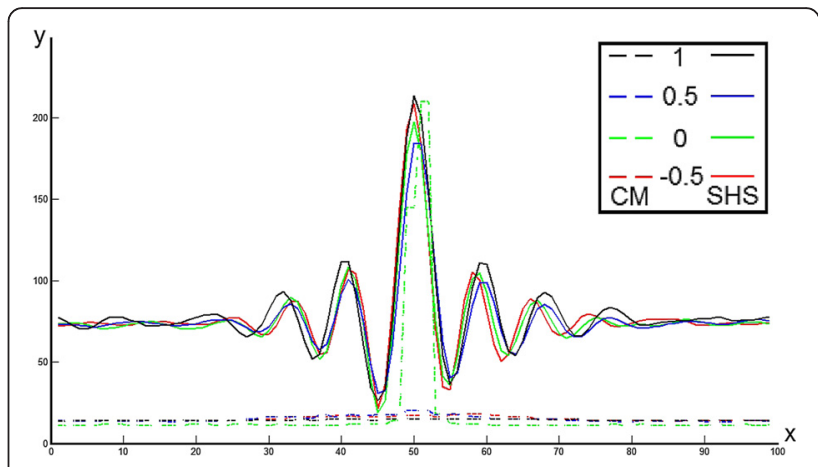

Fig. 10 The cross section of the PSF of the conventional microscope ( $\mathrm{CM},--)$ and the built RBL based holographic setup (SHS, - ), where the target, the $4 \mu \mathrm{m}$ illuminating $(530 \mathrm{~nm})$ spot, is at different distances. $X$ and $Y$ denote the position on the detector and the relative intensity respectively. It can be seen, that the PSF of the SHS does not change significantly with the target distance, but the PSF of the CM changes. The different colours belonging to different target distances as it is shown in the corner

target of distances $(1,0.5,0,-0.5 \mathrm{~mm})$ is presented in Fig. 10. In the experiment, we applied a fibre end with $4 \mu \mathrm{m}$ diameter, which is emitting $530 \mathrm{~nm}$ green light, of a LED.

Because different exposure times have to be used for the holographic and for the conventional microscope, to compare the results I normalised the two measurements. Because holography needs a background for the correct reconstructions to have correct intensity values, the PSFs of the reconstructions have an offset. This decrease the contrast of the reconstructed image. These PSF characteristics that has been reached in the experiments with the built SHS setup also show that the RBL can increase the depth of the observed volume. Although the central spot sizes of the different reconstructions are similar to the conventionally captured spot size (when the target is in focus). The reconstructed rings can bias the resolution in a special way.

\section{Holographic imaging of auto-fluorescent objects}

Let us demonstrate the capabilities of the proposed system. For this purpose, we recorded a self-referenced hologram of an alive filamentous green algae sample. Using a proper emission (longpass colour) filter, the light used for the excitation is filtered. However, this does not increase the coherence length of the emitted fluorescence (Chlorophyll A auto-fluorescent, central wavelength: 680 $\mathrm{nm}$, band with: $\sim 30 \mathrm{~nm}$ ). The reconstruction method was the same as in the experiment detailed above. The fluorescent image, the captured hologram and the reconstruction of the sample can be seen in Fig. 11.

Our measurements show that not only the filamentous green algae but also some other smaller algae fragments are detectable. In conclusion, the RBL based SHS can make an informative holographic image using fluorescent light with $14 \mu \mathrm{m}$ coherence length. 


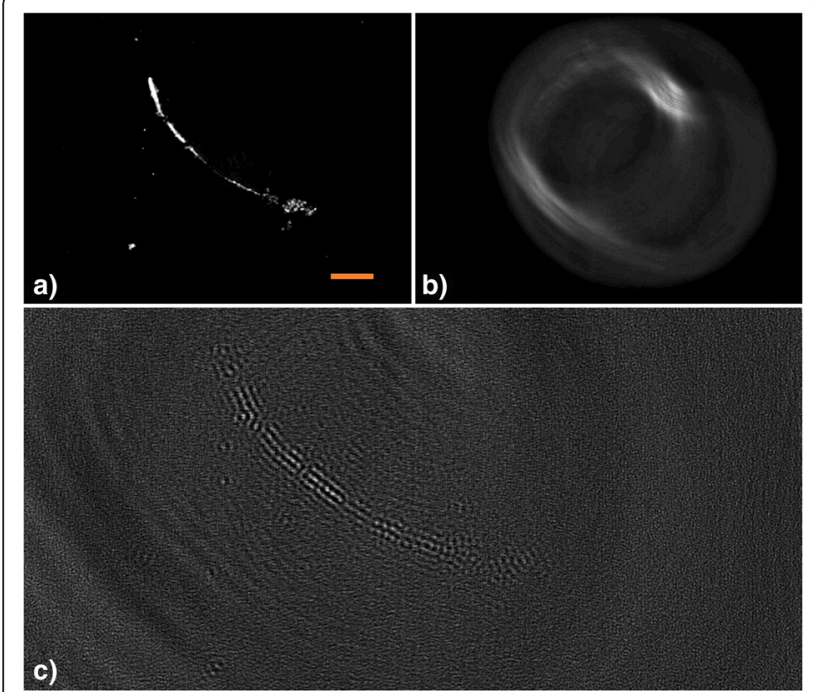

Fig. 11 a Conventional Fluorescent image of a filamentous and some smaller green algae. The orange scale bar is $200 \mu \mathrm{m}$ long. $\mathbf{b}$ The selfreferenced hologram using RBL based SHS. c Reconstructed image. The achieved reconstruction mark the position of the sample objects, it also represents some shape and size information about the target

\section{Discussion}

The main challenge in the design process of the custom Ring-shaped Bifocal Lens (RBL) was to keep the optical path difference of the different beams bellow the coherence length of the fluorescent emission, as close to zero as it is possible. The primary parameter of the design was the thickness difference of the central and the ring-shaped area of the RBL. These are, however, depends on the actual focus parameters of the optical setup. The aperture parameters mostly limit the low-frequency components which will be missing from the self-referenced holograms.

By using a proper band-pass filter, one can increase the coherent length of the emitted fluorescent light, thus the contrast of the fluorescent self-referenced hologram and the contrast of the reconstruction as well. When all the objects emit fluorescent light of the same colour, a band-pass filter can be advantageous; otherwise (such as different algae with different fluorescent emissions) the original RBL should be used without any further filtering.

According to our measurements, the RBL can be efficiently used for self-referenced holography. The most prominent advantages of an RBL is that it is simple, it can be manufactured easily, compact, and the self-referenced architecture based on it is not sensitive to vibrations. Furthermore, its single-shot imaging property makes it applicable to observe freely moving fluorescent objects as well.

However, we must note that the RBL based selfreferenced setups have to deal with some loss of the low-frequency components, which deficiency generates a diffraction-fringe-like noise on the reconstruction. Although this property might limit the imaging property of the RBL based SHS, the exact position of the target object is still determinable. This property can be efficiently utilizable in applications where the 3D position of singular fluorescent point source is to be determined. Our results pave the way for a new kind of implementation of 3D photoactivated localization microscopy [26].

The results and effects connected with the RBL and presented in this article are illustrating the imaging property of the RBL, and can help the further optimizations.

\section{Conclusion}

Building the ring-shaped bifocal lens (RBL) into a common microscope, and making fluorescent measurements with it, we pointed out that the RBL is a useful, and simple device to create stable self-referenced holographic setups for detection, localisation and imaging of fluorescent, freely moving targets of an extended volume.

\section{Acknowledgements}

The author express their thanks to the Ministry of National Development (NFM) for the financial support, granted within the frame of the competitiveness and excellence program, for the project 'Research and development in medical technology for the efficient treatment of cataract', VKSZ_12-1-2013-0080.

The author thanks for the help of Medicontur Ltd. for the fabrication of the custom bifocal lenses.

The author also thanks for the help of Unicam Ltd. for making available special elements of an Olympus microscope for the experiments.

\section{Competing interests}

The authors declare that they have no competing interests.

Received: 4 March 2016 Accepted: 29 April 2016

Published online: 23 June 2016

\section{References}

1. Vörös, L., Mózes, A., Somogyi, B.: "A five-year study of autotrophic winter picoplankton in lake Balaton, Hungary". Aquat. Ecol. 43, 727-734 (2009)

2. Kazemzadeh, F., Jin, C., Yu, M., Amelard R., Haider, S., Saini, S., Emelko, M., Clausi, D.A., Wong, A.:"Multispectral digital holographic microscopy with applications in water quality assessment", (SPIE Optical Engineering + Applications, 957906) (2015).

3. Rosen, J., Brooker, G.: "Digital spatially incoherent Fresnel holography". Opt. Lett. 32, 912-914 (2007)

4. Lohmann, A.W.: "Wavefront reconstruction for incoherent objects". J. Opt. Soc. Am. 55, 1555-1556 (1965)

5. Kiss, M.Z., Nagy, B.J., Lakatos, P., Göröcs, Z., Tőkés, S., Wittner, B., Orzó, L.: "Special multicolor illumination and numerical tilt correction in volumetric digital holographic microscopy". Opt. Express 22, 7559-7573 (2014)

6. Göröcs, Z., Ozcan, A.: "On-chip biomedical imaging". IEEE Rev. Biomed. Eng. 6, 29-46 (2013)

7. Grare, S., Allano, D., Coëtmellec, S., Perret, G., Corbin, F., Brunel, M., Gréhan, G., Lebrun, D.: "Dual-wavelength digital holography for 3D particle image velocimetry: experimental validation". Appl. Optics 55, A49-A53 (2016)

8. Zhang, Y., LĂL', Q., Ge, B.: "Elimination of zero-order diffraction in digital off-axis holography". Opt. Commun. 240, 261-267 (2004)

9. Orzó, L.: "High speed phase retrieval of in-line holograms by the assistance of corresponding off-axis holograms". Opt. Express 23, 16638-16649 (2015)

10. Qin, Y., Zhong, J.: "Quality evaluation of phase reconstruction in led-based digital holography". Chin. Opt. Lett. 7, 1146-1150 (2009)

11. Gabor, D.: "A new microscopic principle". Nature 161, 777-778 (1948)

12. Gabor, D.: "Microscopy by reconstructed wave-fronts". Proc. R. Soc. Lond. A Math. Phys. Sci. 197, 454-487 (1949) 
13. Man, T., Wan, Y., Wu, F., Wang, D.: "Axial localization of fluorescence samples using single-shot self-interference digital holography". In: Digital Holography \& 3-D Imaging Meeting of OSA, DM2A5. (2015)

14. Kim, M.K.: "Full color natural light holographic camera". Opt. Express 21 , 9636-9642 (2013)

15. Mertz, L., Young, N.: "Fresnel transformations of images". SPIE milestone series ms 128, 44-49 (1996)

16. Kiss, M. Zs., Göröcs, Z., Tőkés Sz:" Self-referenced digital holographic microscopy". In: Cellular Nanoscale Networks and Their Applications CNNA, 2012 13th International Workshop on, 1-4, (IEEE, 2012).

17. Wan, Y., Man, T., Wang, D.: "Incoherent off-axis fourier triangular color holography". Opt. Express 22, 8565-8573 (2014)

18. Rosen, J., Brooker, G.: "Fluorescence incoherent color holography". Opt. Express 15, 2244-2250 (2007)

19. Katz, B., Rosen, J., Kelner, R., Brooker, G.: "Enhanced resolution and throughput of fresnel incoherent correlation holography (finch) using dual diffractive lenses on a spatial light modulator (slm)". Opt. Express 20, 9109-9121 (2012)

20. Wang, P.: "Self-interference low-coherent digital holography by engineered volume holographic pupils". In: Digital Holography \& 3-D Imaging Meeting of OSA, DT3A7. (2015)

21. Sirat, G., Psaltis, D.: "Conoscopic holography". Opt. Lett. 10, 4-6 (1985)

22. Rosen, J., Kelner, R:: "Modified lagrange invariants and their role in determining transverse and axial imaging resolutions of self-interference incoherent holographic systems". Opt. Express 22, 29048-29066 (2014)

23. Lin, Y.C., Cheng, C.J., Poon, T.C.: "Optical sectioning with a low-coherence phaseshifting digital holographic microscope". Appl. Optics 50, B25-B30 (2011)

24. M. Zs. Kiss, "A new compact self-referenced holographic setup tested on a fluorescent target", In Digital Holography \& 3-D Imaging Meeting of OSA, DTh1A7 (2015)

25. Shen, F., Wang, A.: "Fast-Fourier-transform based numerical integration method for the Rayleigh-Sommerfeld diffraction formula". Appl. Opt. 45, 1102-1110 (2006)

26. Davis, l.: "The super-resolution revolution". Biochem. Soc. Trans. 37, 1042-1044 (2009)

\section{Submit your manuscript to a SpringerOpen ${ }^{\odot}$ journal and benefit from:}

- Convenient online submission

- Rigorous peer review

- Immediate publication on acceptance

- Open access: articles freely available online

- High visibility within the field

- Retaining the copyright to your article

Submit your next manuscript at $\gg$ springeropen.com 